\title{
Coupled oscillators driven with difference-frequency parametric position feedback
}

William B. Case, Paul J. H. Tjossem, Katherine G. Abrams, and James F. St. Germaine-Fuller

Citation: Journal of Applied Physics 122, 124905 (2017); doi: 10.1063/1.4991887

View online: http://dx.doi.org/10.1063/1.4991887

View Table of Contents: http://aip.scitation.org/toc/jap/122/12

Published by the American Institute of Physics

\section{Articles you may be interested in}

Identification of nasopharyngeal carcinoma from photoluminescence spectra of $3 \mathrm{C}$-SiC nanocrystals

Journal of Applied Physics 122, 124702 (2017); 10.1063/1.4993964

Theory of spin torque nano-oscillator-based phase-locked loop

Journal of Applied Physics 122, 123903 (2017); 10.1063/1.5004117

Exergy in near-field electromagnetic heat transfer

Journal of Applied Physics 122, 124306 (2017); 10.1063/1.5004662

Nonlinear terahertz metamaterials with active electrical control

Applied Physics Letters 111, 121101 (2017); 10.1063/1.4990671

Localized detection of reversal nucleation generated by high moment magnetic nanoparticles using a large-area magnetic sensor

Journal of Applied Physics 122, 123901 (2017); 10.1063/1.5001919

The high temperature impact response of tungsten and chromium

Journal of Applied Physics 122, 115901 (2017); 10.1063/1.4997674

\section{Scilight}

Sharp, quick summaries illuminating the latest physics research

\section{Sign up for FREE!}

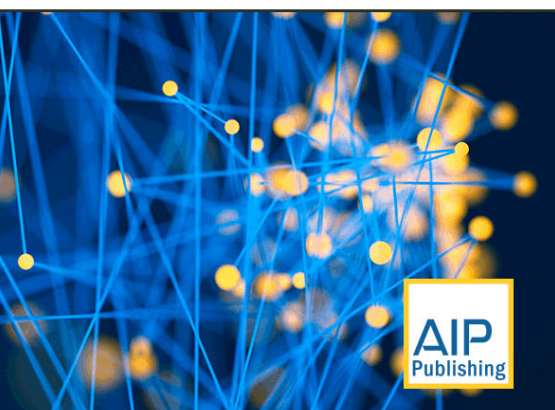




\title{
Coupled oscillators driven with difference-frequency parametric position feedback
}

\author{
William B. Case, ${ }^{\text {a) }}$ Paul J. H. Tjossem, ${ }^{\text {b) }}$ Katherine G. Abrams, ${ }^{\text {c) }}$ \\ and James F. St. Germaine-Fuller ${ }^{\text {d) }}$ \\ Department of Physics, Grinnell College, P.O. Box 805, Grinnell, Iowa 50112, USA
}

(Received 23 June 2017; accepted 14 September 2017; published online 29 September 2017)

\begin{abstract}
We examine theoretically and measure experimentally the instability regions for a pair of parametrically driven mechanical oscillators on an air track. We take the position of the second mass, multiply it by $\cos \left(\omega_{D} t\right)$, and apply it to the first mass. We show that this leads to an instability for both normal modes of the coupled oscillator system when $\omega_{D}$ is close to the difference of the normal mode frequencies. Such an instability mechanism is of special interest because the input frequency, $\omega_{D}$, can be much lower than the output frequencies of the system. In addition, we consider the case where $\cos \left(\omega_{D} t\right)$ is replaced by a negative constant, which also leads to instability, but at a single frequency. This study of the difference frequency oscillator system was motivated by the QASER system described in Svidzinsky, Yuan and Scully [Phys. Rev. X 3, 041001 (2013)]. Published by AIP Publishing. https://doi.org/10.1063/1.4991887
\end{abstract}

\section{INTRODUCTION}

A simple driven parametric oscillator consists of a single harmonic oscillator of natural frequency $\omega_{0}$ driven at frequency $\omega_{D}$ via a term of the form $\cos \left(\omega_{D} t\right)$ times the independent variable describing the oscillator. Such a system leads directly to the Mathieu equation ${ }^{1-4}$ and is known to exhibit instabilities when

$$
\omega_{d}=\frac{2 \omega_{0}}{n} \quad n=1,2,3, \ldots
$$

where $\omega_{0}$ is the natural frequency of the harmonic oscillator. It must be noted that, except for $n=1$, these instabilities tend to be weak. Thus, although the system can be driven at subharmonic frequencies, the driving source must typically deliver a frequency comparable to, and indeed twice, the natural frequency of the system.

We study a pair of coupled harmonic oscillators where oscillator 1 has an added term of the form $\cos \left(\omega_{D} t\right)$ times the independent variable describing oscillator 2 . Unlike the simple parametric oscillator, this system is unstable when $\omega_{D}$ is equal to the difference of the frequencies of the normal modes of the two-oscillator system. Thus the system can be driven effectively by a frequency much lower than either of the natural frequencies of the original harmonic oscillators. This work was motivated by the work of Svidzinsky, Yuan and Scully ${ }^{5,6}$ for a laser system which they call the QASER (Quantum Amplification of Superradiant Emission of Radiation).

In this paper we examine a particularly simple mechanical arrangement, but analogous electrical and optical systems

\footnotetext{
a)case@grinnell.edu

b)tjossem@grinnell.edu

c)abramska@grinnell.edu

${ }^{d)}$ Present address: Department of Physics, California Institute of Technology, 1200 East California Boulevard, Pasadena, California 91125, USA. Electronic mail: jstgerma@caltech.edu.
}

can be of this form. For example, coupled Micro Electro Mechanical Systems (MEMS) are under active study for parametric amplification, coherent control, and quantum entanglement. $^{7-9}$ The system here is taken to be linear, which of course is an approximation. As the system's amplitude grows, nonlinear effects will cause saturation, ending the growth.

In Sec. II, we develop a first order theory of the coupled oscillator system described above, along with a related configuration where the $\cos \left(\omega_{D} t\right)$ factor is replaced by a negative constant. Section III describes the experiment and the experimental results are given in Sec. IV. A discussion of the source of the energy is given in Sec. V. Final conclusions are given in Sec. VI.

\section{THEORY}

A pair of identical mass-spring oscillators $(m, k)$ is weakly coupled with a central spring $\left(k_{c}<k\right)$, as shown in Fig. 1. The positions of the two masses are labeled $x_{1}$ for the left mass and $x_{2}$ for the right mass. An external force is applied to the left-hand spring by driving a piston with signal $s f(t) x_{2}$, where $s$ is a scale factor between $x_{2}$ and the piston position, and $f(t)$ is either a harmonic function or a negative constant. The equations of motion are thus

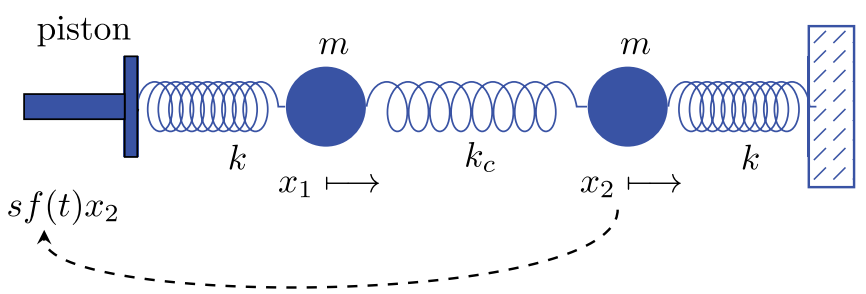

FIG. 1. A pair of equal masses is weakly coupled with central spring $k_{c}<k$. Held rigidly on the right side, the left side is driven with the product signal $s f(t) x_{2}$, where $s$ represents a scale factor between $x_{2}$ motion and the piston motion, and $f(t)$ is an external frequency. The dashed line represents the feedback path from $x_{2}$ to the piston. 


$$
\begin{gathered}
m \ddot{x}_{1}=-k x_{1}+k_{c}\left(x_{2}-x_{1}\right)-r \dot{x}_{1}+k s f(t) x_{2}, \\
m \ddot{x}_{2}=-k x_{2}-k_{c}\left(x_{2}-x_{1}\right)-r \dot{x}_{2},
\end{gathered}
$$

where the coefficient of damping is given by $r$. We simplify these equations by defining the following variables:

$$
\begin{gathered}
\omega_{0}^{2}=\frac{k+k_{c}}{m}, \\
\alpha=\frac{k_{c}}{m}, \\
\beta=\frac{k s}{m}, \\
\eta=\frac{r}{m} .
\end{gathered}
$$

Our equations are now

$$
\begin{gathered}
\ddot{x}_{1}+\omega_{0}^{2} x_{1}+\eta \dot{x}_{1}=\alpha x_{2}+\beta x_{2} f(t), \\
\ddot{x}_{2}+\omega_{0}^{2} x_{2}+\eta \dot{x}_{2}=\alpha x_{1} .
\end{gathered}
$$

\section{A. Constant negative multiplier, $\beta f(t)=-\beta_{0}$}

If $f(t)$ in Eq. (4a) is a negative constant such that $\beta f(t)=-\beta_{0}$, the equations of motion become

$$
\begin{gathered}
\ddot{x}_{1}+\omega_{0}^{2} x_{1}+\eta \dot{x}_{1}=\alpha x_{2}-\beta_{0} x_{2}, \\
\ddot{x}_{2}+\omega_{0}^{2} x_{2}+\eta \dot{x}_{2}=\alpha x_{1} .
\end{gathered}
$$

We seek solutions of the form

$$
\begin{aligned}
& x_{1}=A e^{i \omega_{0} t}, \\
& x_{2}=B e^{i \omega_{0} t},
\end{aligned}
$$

where $A$ and $B$ vary slowly in time. Substituting these last two equations and their time derivatives into Eqs. (5a) and (5b), and neglecting $\ddot{A}$ and $\ddot{B}$ gives

$$
\begin{gathered}
(2 \dot{A}+\eta A) i \omega_{0}+\eta \dot{A}=\left(\alpha-\beta_{0}\right) B, \\
(2 \dot{B}+\eta B) i \omega_{0}+\eta \dot{B}=\alpha A .
\end{gathered}
$$

We now look for exponentially growing (or decaying) solutions by letting $A$ and $B$ be of the form

$$
\begin{aligned}
& A(t)=a e^{\lambda t}, \\
& B(t)=b e^{\lambda t},
\end{aligned}
$$

with $a$ and $b$ constants. In keeping with our earlier neglect of $\ddot{A}$ and $\ddot{B}$, we require $\lambda \ll \omega_{0}$. Substituting Eqs. (8) into Eqs. (7) and solving the resulting quadratic gives, in the limit of weak damping $\left(\eta \ll \omega_{0}\right)$, growth rate

$$
\lambda=-\frac{\eta}{2} \pm \frac{1}{2 \omega_{0}} \sqrt{\alpha\left(\beta_{0}-\alpha\right)} .
$$

This equation yields a positive growth rate if

$$
\beta_{0} \geq \frac{\eta^{2} \omega_{0}^{2}}{\alpha}+\alpha \approx \alpha
$$

where the last approximation again assumes small damping. Above this threshold the masses oscillate $\pi / 2$ out of phase, with the amplitude ratio

$$
\frac{x_{2}}{x_{1}}=\frac{\alpha}{\omega_{0}(2 \lambda+\eta)}=\sqrt{\frac{\alpha}{\beta_{0}-\alpha}} .
$$

The masses oscillate at a single frequency $\omega_{0}$ that lies between the two normal mode frequencies of the coupled oscillators $\omega_{1}$ and $\omega_{2}$ [explicitly expressed below in Eqs. (14c) and (14d)], because the coupling spring stretches during each oscillation, but only half as much as for the $\omega_{2}$ asymmetric stretch. For the particular value of $\beta_{0}=2 \alpha$, the two masses oscillate with identical amplitudes.

\section{B. External multiplying frequency}

We now consider an external force applied to the lefthand spring by letting $f(t)=\cos \left(\omega_{D} t\right)$ in Eq. (4a), where $\omega_{D}$ represents an adjustable driving frequency. The equations of motion now become

$$
\begin{gathered}
\ddot{x}_{1}+\omega_{0}^{2} x_{1}+\eta \dot{x}_{1}=\alpha x_{2}+\beta \cos \left(\omega_{D} t\right) x_{2}, \\
\ddot{x}_{2}+\omega_{0}^{2} x_{2}+\eta \dot{x}_{2}=\alpha x_{1} .
\end{gathered}
$$

Adding and subtracting these equations gives

$$
\begin{aligned}
& \ddot{x}_{+}+\omega_{1}^{2} x_{+}+\eta \dot{x}_{+}=\frac{\beta}{2} \cos \left(\omega_{D} t\right)\left(x_{+}-x_{-}\right), \\
& \ddot{x}_{-}+\omega_{2}^{2} x_{-}+\eta \dot{x}_{-}=\frac{\beta}{2} \cos \left(\omega_{D} t\right)\left(x_{+}-x_{-}\right),
\end{aligned}
$$

where

$$
\begin{aligned}
& x_{+}=x_{1}+x_{2}, \\
& x_{-}=x_{1}-x_{2}, \\
& \omega_{1}^{2}=\omega_{0}^{2}-\alpha, \\
& \omega_{2}^{2}=\omega_{0}^{2}+\alpha .
\end{aligned}
$$

These last two equations are the usual normal mode frequencies $\omega_{1}$ and $\omega_{2}$ of the coupled oscillators, corresponding to symmetric and asymmetric stretching modes, respectively. We define the difference frequency between the normal modes as

$$
\omega_{21} \equiv \omega_{2}-\omega_{1} .
$$

The system is again unstable but now for

$$
\omega_{D} \approx \omega_{21}
$$

because the product term $\beta \cos \left(\omega_{D} t\right) x_{2}$ in Eq. (12a) will mix the two frequencies into each other. We thus seek a solution of the form

$$
\begin{aligned}
& x_{+}=A e^{i \omega_{1} t}, \\
& x_{-}=B e^{i \omega_{2} t},
\end{aligned}
$$

where again $A$ and $B$ vary slowly with time. Setting $\omega_{D}=\omega_{21}$, and substituting into Eqs. (13a) and (13b), gives 


$$
\begin{gathered}
2 i \omega_{1} \dot{A}+i \omega_{1} \eta A=-\frac{\beta}{4} B, \\
2 i \omega_{2} \dot{B}+i \omega_{2} \eta B=\frac{\beta}{4} A,
\end{gathered}
$$

where $\ddot{A}, \ddot{B}, \eta \dot{A}, \eta \dot{B}$, and non-resonant terms such as $A e^{i\left(2 \omega_{1}-\omega_{2}\right) t}$ have been neglected. Again substituting for $A$ and $B$ via Eqs. (8a) and (8b), and solving for $\lambda$ yields a growth rate at $\omega_{D}=\omega_{21}$ of

$$
\lambda=-\frac{\eta}{2} \pm \frac{\beta}{8 \sqrt{\omega_{1} \omega_{2}}} .
$$

In order to achieve positive growth $\frac{\beta}{8 \sqrt{\omega_{1} \omega_{2}}}$ must exceed the damping rate $\eta / 2$ when $\omega_{D}=\omega_{21}$. In the Appendix, we consider the case where $\omega_{D}$ is close but not exactly equal to $\omega_{21}$ in order to determine the width and shape of the instability.

\section{EXPERIMENTAL ARRANGEMENT}

Two $m=134 \mathrm{~g}$ gliders are placed on an air track as shown in Fig. 2. A linear actuator (Servo City HDA2-2) drives glider 1 via spring $k$. A weak coupling spring $k_{c}$ connects glider 1 to glider 2, which is attached to a fixed support via a second spring $k$. A quadrature linear encoder (Avago HEDS9200-360) is fixed over glider 2 upon which is mounted a 360 line pair per inch codestrip (Laserlab, Inc.) to provide contactless relative position readings of $x_{2}$ over the entire extent of the $90 \mathrm{~mm}$-long glider. The $x_{2}$ pulses are decoded in real time by a microcontroller (Microchip PIC HPC Explorer) via interrupts on all four logic edges, permitting relative position readings at rates in excess of $20 \mathrm{kHz}$ with a precision of $18 \mu \mathrm{m}$. The maximal excursion of the $x_{2}$ glider amounts to an amplitude of $36.1 \mathrm{~mm}$. The gliders are started from rest at equilibrium, and the zero position is reset to 2048 counts (half scale on 12-bits) by averaging the small excursions about equilibrium prior to powering the piston. The motion for each

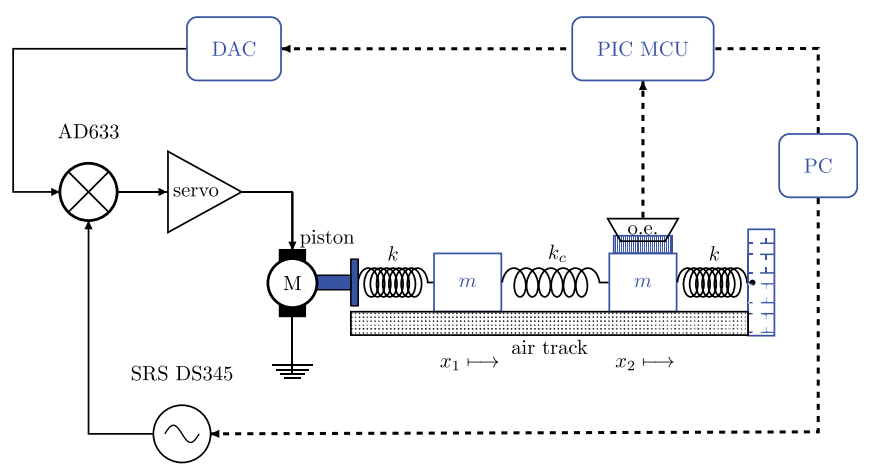

FIG. 2. A pair of equal mass gliders held together with three springs freely oscillate on an air track. The two outer springs are identical, and the coupling is via a weak central spring $k_{c}<k$. A PIC microcontroller monitors position $x_{2}$ via an optical encoder (o.e.) and a linear code strip, and then outputs the analog position via a digital-to-analog converter. The analog $x_{2}$ position signal and an external signal from a function generator are multiplied together via the AD633 to provide positioning input to the servo driver that moves the piston. A separate real-time control loop (not shown) monitors the piston position and ensures that the piston responds linearly to the servo input. The digital signal paths are shown as dashed lines; the analog signals are shown as solid lines.
TABLE I. AD633 analog multiplier inputs and feedback modes: $\mathrm{W}=(\mathrm{X} 1$ $\mathrm{X} 2) \times(\mathrm{Y} 1-\mathrm{Y} 2) /(10 \mathrm{~V})+\mathrm{Z}$.

\begin{tabular}{lcccccc}
\hline \hline I/O mode & $\mathrm{X} 1$ & $\mathrm{X} 2$ & $\mathrm{Y} 1$ & $\mathrm{Y} 2$ & $\mathrm{Z}$ & $(\mathrm{W}-\mathrm{Z}) \times 10 \mathrm{~V}$ \\
\hline Product & $x_{2}$ & $2.5 \mathrm{~V}$ & $s \cos \left(\omega_{D} t\right)$ & 0.0 & Fixed & $s x_{2} \cos \left(\omega_{D} t\right)$ \\
Self & $x_{2}$ & $2.5 \mathrm{~V}$ & $s \mathrm{~V}_{1}$ & 0.0 & Fixed & $s x_{2} \mathrm{~V}_{1}$ \\
None & $\mathrm{V}_{0}$ & $2.5 \mathrm{~V}$ & $s \cos \left(\omega_{D} t\right)$ & 0.0 & Fixed & $s \mathrm{~V}_{0} \cos \left(\omega_{D} t\right)$ \\
\hline \hline
\end{tabular}

experiment is initiated by noise in the position readout. The PIC microcontroller is connected to a 3-wire serial 12-bit digital to an analog converter (DAC) (Microchip MCP4922) to give a unipolar $0-5 \mathrm{~V}$ representation of position $x_{2}$. Upon leaving the DAC, all subsequent processing involves analog signals. The analog $x_{2}$ position reading is offset by $-2.5 \mathrm{~V}$ at one of the multiplying inputs of an analog multiplier (Analog Devices AD633) in order to present a zero average value to the multiplier. The other multiplicand input controls the nature of the feedback, and is set at either a fixed voltage or a pure sine wave at a driving frequency $\omega_{D}$. The sine wave or offset voltage is supplied by a computer-controlled function generator (SRS DS345). Finally, the product signal is added to a fixed offset voltage to extend the linear actuator; this signal becomes the input voltage to the servo motor controller (Servo City, Pololu JRK) that drives the actuator. A second linear encoder/ DAC pair is used solely to close the motor feedback around the linear encoder position, ensuring near-sinusoidal motion without gear backlash. Depending upon the absence or presence of $x_{2}$ position feedback at the multiplier, this arrangement can behave as an ordinary end-driven coupled oscillator or as a parametrically driven system, with position $x_{2}(t)$ providing the varying parameter. The feedback modes are summarized in Table I. A laboratory PC collects the $x_{2}$ encoder counts at $100 \mathrm{~Hz}$, the same rate at which the serial DAC voltage is sent to the multiplier, and monitors the synchronization pulse provided by the function generator.

\section{RESULTS}

Measurements of the normal mode frequencies of the coupled oscillators yielded $\omega_{1}=2.824 \mathrm{rad} / \mathrm{s}$ and $\omega_{2}=3.538 \mathrm{rad} / \mathrm{s}$, putting the difference frequency $\omega_{21}=0.714 \mathrm{rad} / \mathrm{s}, \omega_{0}=$ $3.201 \mathrm{rad} / \mathrm{s}$, and $\alpha=2.271 \mathrm{~s}^{-2}$. The damping rate $\eta / 2$ was measured to be $5.65 \times 10^{-3} s^{-1}$. The experimental parameters are summarized in Table II.

\section{A. Fixed negative multiplier $-\beta_{0}$}

Figure 3 shows the position $x_{2}$ vs. time when the external input to the multiplier is supplied with a sufficiently large constant negative voltage, i.e., when $\beta_{0}$ overcomes the coupling constant $\alpha$. The amplitude grows exponentially at a single frequency $\omega_{0}$. The two masses oscillate in quadrature, typically with different amplitudes, depending upon the value of the external voltage, i.e., the gain. The first mass

TABLE II. Experimental parameters for this study.

\begin{tabular}{cccccc}
\hline \hline$\omega_{1}\left(\mathrm{~s}^{-1}\right)$ & $\omega_{2}$ & $\omega_{0}$ & $\omega_{21}$ & $\eta / 2$ & $\alpha\left(\mathrm{s}^{-2}\right)$ \\
\hline 2.82 & 3.54 & 3.20 & 0.714 & $5.65 \times 10^{-3}$ & 2.27 \\
\hline \hline
\end{tabular}




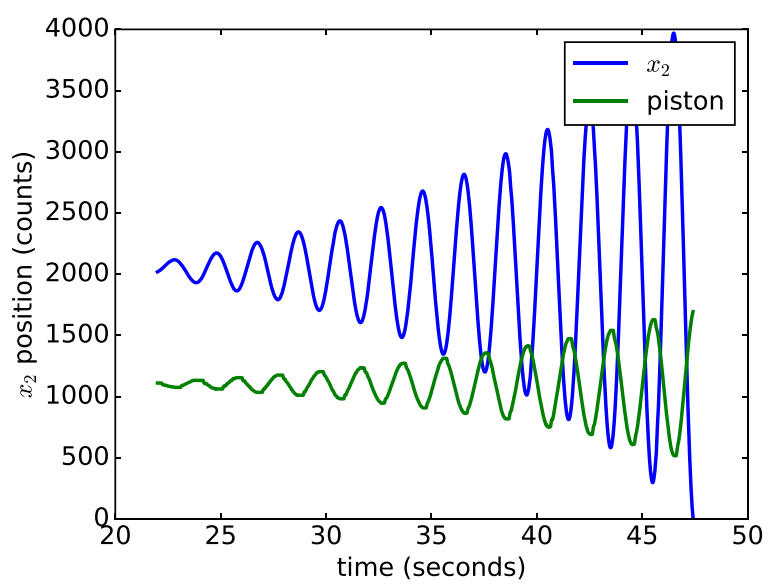

FIG. 3. Experimental position $x_{2} v s$. time, along with the piston position, for fixed negative multiplier above the threshold given in Eq. (9). Here, $\beta_{0}=2.39$, which exceeds $\alpha=2.27$. The negative constant gain ensures that the piston is $180^{\circ}$ out of phase with the $x_{2}$ position. Each count represents a distance of $18 \mu \mathrm{m}$.

(not shown) oscillates at $90^{\circ}$ out of phase with the piston, and the second mass oscillates a further $90^{\circ}$ out of phase with the first mass. The feedback signal $x_{2}$ is thus $180^{\circ}$ relative to the piston; when inverted by the multiplier's fixed negative constant and returned to the piston's servo drive motor the overall feedback is positive, allowing selfsustaining oscillation, if the magnitude of the loop gain exceeds unity, at the single frequency $\omega_{0}$ as shown in Fig. 4. The present system thus forms a phase shift oscillator, ${ }^{10}$ commonly implemented with three or four RC sections, but in this case the analogous electronic circuit would be formed from a pair of LC sections. ${ }^{11}$ Figure 5 examines the threshold characteristics of this fixed negative multiplier case. For $\beta_{0}<\alpha$ there is no growth in $x_{2}$, as predicted from Eq. (10). As $\beta_{0}$ exceeds $\alpha$ and overcomes the damping, $\lambda$ increases with $\beta_{0}$ in accordance with Eq. (9), in complete agreement with the small-signal theory developed in Sec. II A.

\section{B. External frequency $\omega_{D} \approx \omega_{21}$}

Figure 6 shows the position $x_{2}$ vs. time when the AD633 multiplies the position $x_{2}$ with an external signal $\beta \cos \left(\omega_{D} t\right)$

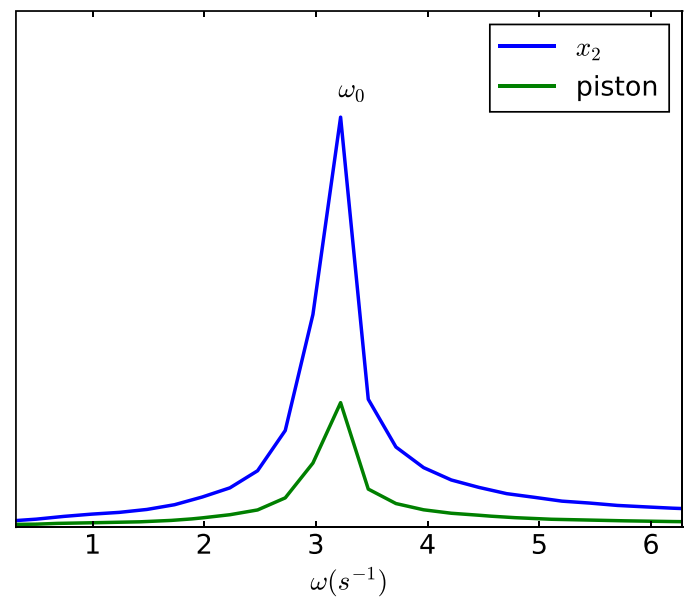

FIG. 4. Fourier transform of the $x_{2}$ and piston data taken with fixed negative multiplier $-\beta_{0}$ yield a single frequency $\omega_{0}$.

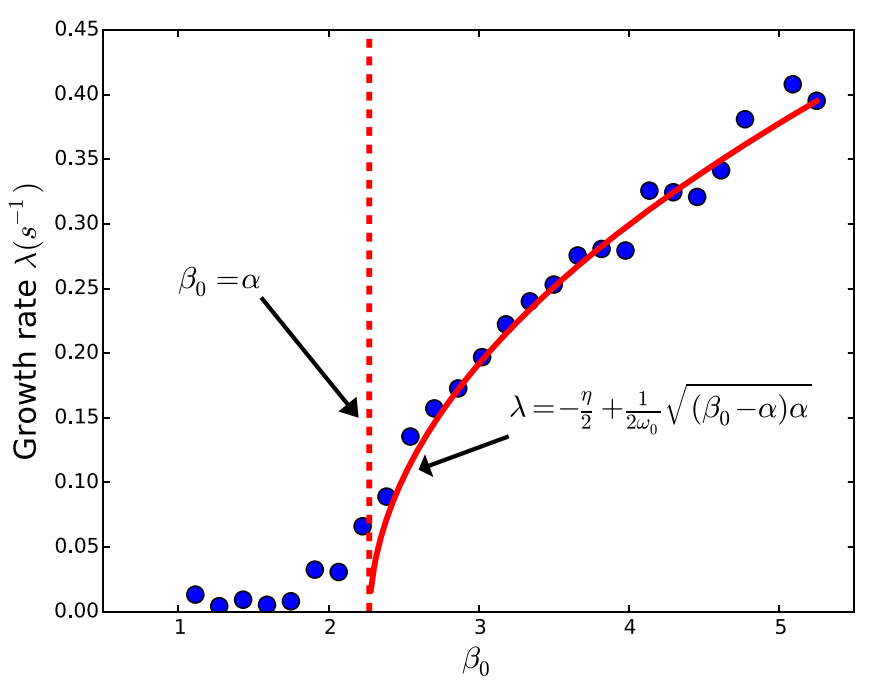

FIG. 5. Measured growth rate as a function of $\beta_{0}$ for the fixed negative multiplier case. Below $\beta_{0}=\alpha$ there is no growth, as predicted in Eq. (10). Above this threshold the growth increases according to Eq. (9), which is shown as the solid curve, plotted using data from Table II, with no adjustable parameters.

before driving the piston. If $\omega_{D}$ is near the difference frequency $\omega_{21}=\omega_{2}-\omega_{1}$, and $\beta$ is sufficiently large to overcome the damping, the amplitude of $x_{2}$ grows exponentially in both modes, as shown in the Fourier transform of Fig. 7. The FFT spectrum of the piston position exhibits the characteristic sidebands of double sideband suppressed carrier (DSB-SC) mixing, ${ }^{12}$ a form of AM modulation that results from pure multiplication without the offset, along with the nearly suppressed carrier at $\omega_{D}=\omega_{21}$. In this case, the DSB$\mathrm{SC}$ modulation is applied in an unorthodox fashion, in that the modulating bandwidth $\omega_{2}$ exceeds the frequency of the carrier, $\omega_{D}$. The highest frequency needed to drive the piston is $\omega_{2}+\left(\omega_{2}-\omega_{1}\right)=2 \omega_{2}-\omega_{1}$, as shown in the FFT spectrum of the piston.

Figure 8 shows the measured growth rate as a function of detuning for several values of the driver strength $\beta$,

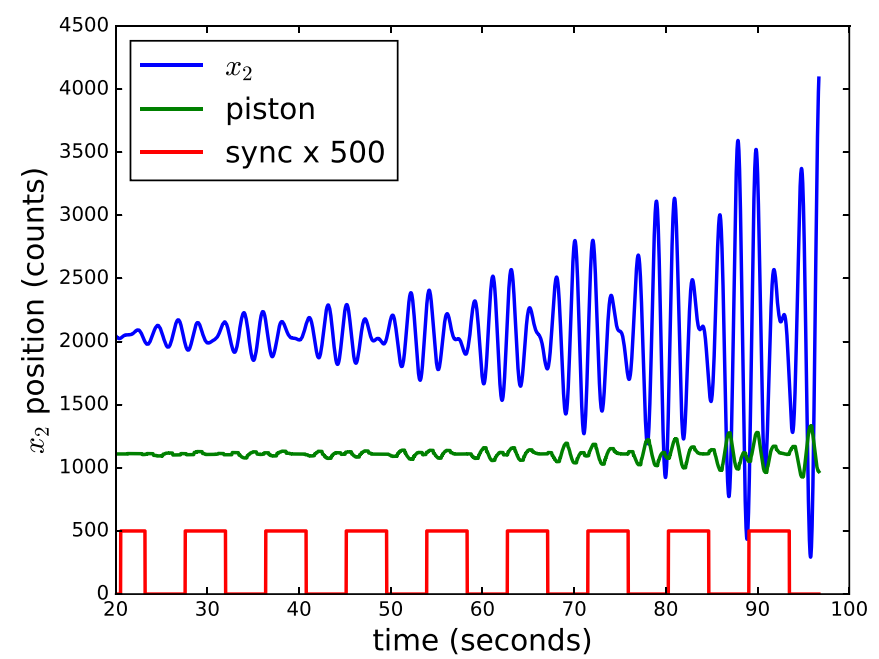

FIG. 6. Experimental position $x_{2}$ and piston position $v s$. time for external frequency $\omega_{D}$ near $\left(\omega_{2}-\omega_{1}\right)$, and $\beta=1.11$. Shown also is the low difference frequency $\omega_{D}$ synchronization pulse from the external function generator. 


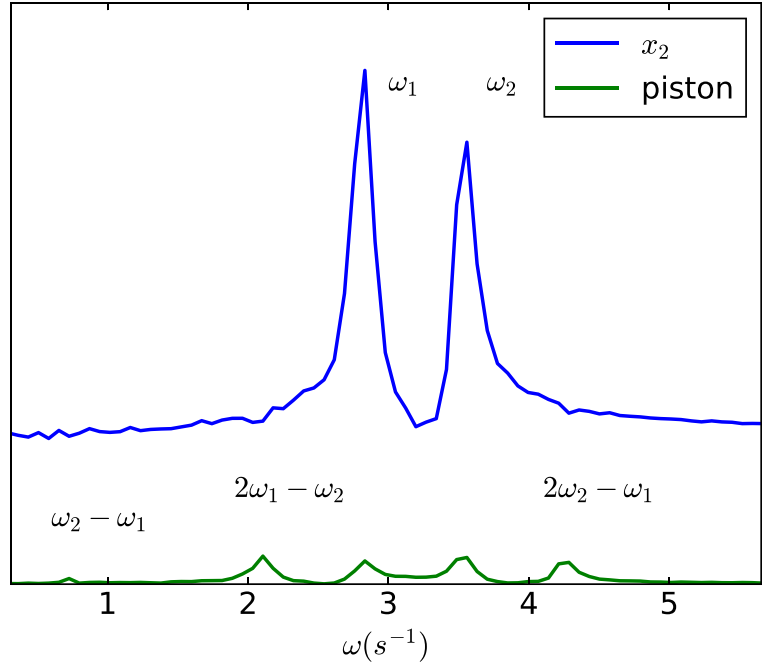

FIG. 7. The Fourier transform of $x_{2} v s$. time data (upper trace) taken near $\omega_{D}=\omega_{21}$, showing the two normal modes at $\omega_{1}$ and $\omega_{2}$. The corresponding piston data (lower trace) exhibit characteristic sidebands of DSB-SC modulation at $2 \omega_{1}-\omega_{2}$ and $2 \omega_{2}-\omega_{1}$, and the partially suppressed carrier at $\omega_{21}=\omega_{2}-\omega_{1}$. The traces are offset vertically for clarity.

plotted along with the analytical expression for the detuning as developed in the Appendix. The growth rates are proportional to $\beta$ as predicted in Eq. (19). The small feature near $\omega_{21} / 2$ is the first Arnold tongue, ${ }^{13}$ visible only at the highest values of $\beta$.

\section{ENERGY CONSIDERATIONS}

The exponential growth exhibited by these systems requires a source of power supplied to glider $x_{1}$ via the product of the force of the piston times the velocity of $m_{1}$,

$$
P=F \cdot v_{1}=k s f(t) x_{2} \dot{x}_{1} .
$$

This power is supplied to the linear actuator via the servo driver after the multiplication of $x_{2}$ by $f(t)$; the power sourced

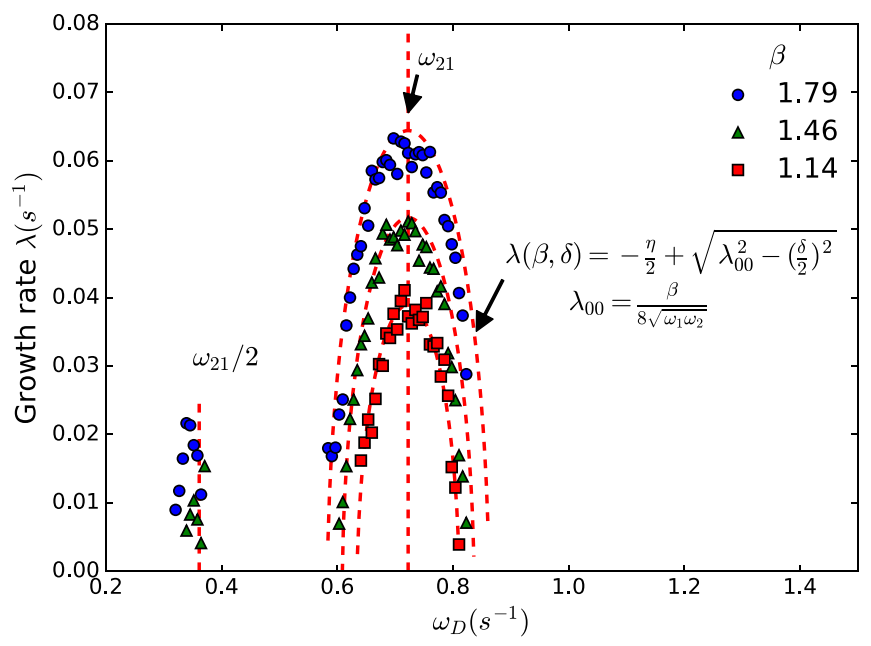

FIG. 8. Measured growth rates as a function of external driver frequency $\omega_{D}$ for various $\beta \cos \left(\omega_{D} t\right)$, along with the analytical expression derived in the Appendix. The small and narrow feature just to the red side of $\omega_{21} / 2$ is an Arnold tongue. The dashed curves are constructed using the measured parameters from Table II, the driver amplitude $\beta$, and the detuning frequency $\delta$, with no adjustable parameters. by either input is negligibly small by comparison. This locus of power delivery may appear to disqualify the present system as a model of the QASER or similar mechanisms, wherein the low-frequency external source $f(t)$ provides the majority of the power. Yet by a simple gedanken modification of the system the energy source can be thought of as a strong signal proportional to $x_{2}$ or equally as a strong signal arising from $f(t)$.

For the latter case, consider a potentiometer in which the strong signal $f(t)$ is applied across the entire resistor. Assume that the signal is sufficiently strong that the current is given by $I_{0} f(t)$, where $I_{0}$ is independent of time. The location of the slider is to be determined by the weak $x_{2}$ signal. The output voltage is now given by $b I_{0} f(t) x_{2}$, where $b$ is a constant. This final signal is fed to a linear solenoid which replaces our actuator to feed energy into the system. In this case, the primary source of the power is the strong $f(t)$ signal. Alternatively, making $x_{2}$ the strong signal and $f(t)$ the weak can be achieved by reversing the roles of the two signals in our gedanken experiment. In either case, the behavior of the system would be identical to the results presented here.

\section{CONCLUSIONS}

We have analyzed and constructed a novel mechanical system of two coupled oscillators wherein the position of one is monitored and used to determine a force that is applied to the other. This parametric applied force is either a negative constant times the position or a harmonic function times the position. Both cases produce regions where the oscillations are unstable. In the first case, the system is unstable when the strength of the constant feedback exceeds the coupling strength plus the damping, creating a form of phase-shift oscillator with overall positive feedback. The second case results in instability when the driver frequency matches the difference between the two normal mode frequencies. The onset of exponential growth in this second case is at considerably lower values of the product signal; the applied product signal needs merely to exceed the damping. The measured growth rates and the width of the instability region match our first-order theory.

\section{ACKNOWLEDGMENTS}

The authors would like to thank Professors Wolfgang Schleich and Marlan Scully for fruitful and spirited discussions on the QASER mechanism and the topic of difference frequency oscillations.

\section{APPENDIX: WIDTH OF THE DIFFERENCE FREQUENCY INSTABILITY}

In this section, we examine the width of the instability about $\omega_{D}=\omega_{21} \cdot{ }^{14}$ Setting $\omega_{D}=\omega_{2}-\omega_{1}+\delta$ in Eqs. (13a) and (13b), we seek solutions of the form

$$
\begin{aligned}
& x_{+}=A e^{i\left(\omega_{1}-\frac{\delta}{2}\right) t}, \\
& x_{-}=B e^{i\left(\omega_{2}+\frac{\delta}{2}\right) t},
\end{aligned}
$$


where $A$ and $B$ are again assumed to vary slowly with time and $\delta$ is small. With this substitution, we find

$$
\begin{aligned}
& 2 i \omega_{1} \dot{A}+\omega_{1}(\delta+i \eta) A=-\frac{\beta}{4} B \\
& 2 i \omega_{2} \dot{B}+\omega_{2}(-\delta+i \eta) B=\frac{\beta}{4} A .
\end{aligned}
$$

In obtaining these equations, we have assumed $\delta$ and $\eta$ are much smaller that the $\omega$ 's, and have neglected non-resonant terms. Finally we assume exponential solutions as in Eqs. (8a) and (8b) and solve for $\lambda$, yielding

$$
\lambda=-\frac{\eta}{2} \pm \sqrt{\left(\frac{\beta}{8}\right)^{2} \frac{1}{\omega_{1} \omega_{2}}-\left(\frac{\delta}{2}\right)^{2}} .
$$

From this expression, we see that the maximum growth rate is

$$
\lambda=-\frac{\eta}{2}+\frac{\beta}{8 \sqrt{\omega_{1} \omega_{2}}},
$$

reached when $\delta=0$, as previously noted in Eq. (19); in order to achieve positive growth $\frac{\beta}{8 \sqrt{\omega_{1} \omega_{2}}}$ must be greater than $\eta / 2$, as found following Eq. (19). In the absence of damping, we define the maximum undamped growth rate as

$$
\lambda_{00} \equiv \frac{\beta}{8 \sqrt{\omega_{1} \omega_{2}}} .
$$

The growth rate may then be written as

$$
\lambda=-\frac{\eta}{2}+\sqrt{\lambda_{00}^{2}-\left(\frac{\delta}{2}\right)^{2}} .
$$

The second term forms an ellipse whose major axis lies along $\delta=0$ and has length $2 \lambda_{00}$.

${ }^{1}$ N. W. McLachlan, Theory and Application of Mathieu Functions (Dover, New York, 1947).

${ }^{2}$ L. D. Landau and E. M. Lifshitz, Mechanics, 3rd ed. (Elsevier Butterworth-Heinemann, 1976).

${ }^{3}$ W. B. Case, Am. J. Phys. 48, 218 (1980).

${ }^{4}$ J. J. Sanmartín, Am. J. Phys. 52, 937 (1984).

${ }^{5}$ A. A. Svidzinsky, L. Yuan, and M. O. Scully, Phys. Rev. X 3, 041001 (2013).

${ }^{6}$ G. Chen, J. Tian, B. Bin-Mohsin, R. Nessler, A. A. Svidzinsky, and M. O. Scully, Phys. Scr. 91, 073004 (2016).

${ }^{7}$ H. Okamoto, A. Gourgout, C.-Y. Chang, K. Onomitsu, I. Mahboob, E. Y. Chang, and H. Yamaguchi, Nat. Phys. 9, 480 (2013).

${ }^{8}$ S.-B. Shim, M. Imboden, and P. Mohanty, Science 316, 95 (2007). http:// science.sciencemag.org/content/316/5821/95.full.pdf.

${ }^{9}$ These MEMS devices are usually excited via non-linear parametric driving of the coupling capacitance, near twice the natural frequency of either oscillator.

${ }^{10}$ T. L. Floyd, Electronic Devices: Electron Flow Version, 9th ed. (Pearson, 2012), p. 815.

${ }^{11}$ H. F. Olson, Dynamical Analogies (Van Nostrand, New York, 1943), p. 48.

${ }^{12} \mathrm{P}$. Horowitz and W. Hill, The Art of Electronics, 2nd ed. (Cambridge, 1989), p. 897.

${ }^{13}$ K. Staliunas, C. Hang, and V. V. Konotop, Phys. Rev. A 88, 023846 (2013).

${ }^{14}$ For a more detailed treatment of this instability region, see Ref. 6. 